\title{
New Convergence Definitions for Sequences of Sets
}

\author{
Ömer Kişi ${ }^{1}$ and Fatih Nuray ${ }^{1,2}$ \\ ${ }^{1}$ Faculty of Education, Mathematics Education Department, Cumhuriyet University, Sivas, Turkey \\ ${ }^{2}$ Department of Mathematics, Faculty of Science and Literature, Afyon Kocatepe University, Afyonkarahisar, Turkey
}

Correspondence should be addressed to Fatih Nuray; fnuray@aku.edu.tr

Received 14 May 2013; Accepted 26 September 2013

Academic Editor: Svatoslav Staněk

Copyright (C) 2013 Ö. Kişi and F. Nuray. This is an open access article distributed under the Creative Commons Attribution License, which permits unrestricted use, distribution, and reproduction in any medium, provided the original work is properly cited.

Several notions of convergence for subsets of metric space appear in the literature. In this paper, we define Wijsman I-convergence and Wijsman $I^{*}$-convergence for sequences of sets and establish some basic theorems. Furthermore, we introduce the concepts of Wijsman I-Cauchy sequence and Wijsman $I^{*}$-Cauchy sequence and then study their certain properties.

\section{Introduction and Background}

The concept of convergence of sequences of points has been extended by several authors (see [1-9]) to the concept of convergence of sequences of sets. The one of these such extensions that we will consider in this paper is Wijsman convergence. We will define $I$-convergence for sequences of sets and establish some basic results regarding these notions.

Let us start with fundamental definitions from the literature. The natural density of a set $K$ of positive integers is defined by

$$
\delta(K):=\lim _{n \rightarrow \infty} \frac{1}{n}|\{k \leq n: k \in K\}|,
$$

where $|k \leq n: k \in K|$ denotes the number of elements of $K$ not exceeding $n([10])$.

Statistical convergence of sequences of points was introduced by Fast [11]. In [12], Schoenberg established some basic properties of statistical convergence and also studied the concept as a summability method.

A number sequence $x=\left(x_{k}\right)$ is said to be statistically convergent to the number $\xi$ if, for every $\varepsilon>0$,

$$
\lim _{n \rightarrow \infty} \frac{1}{n}\left|\left\{k \leq n:\left|x_{k}-\xi\right| \geq \varepsilon\right\}\right|=0 .
$$

In this case, we write $s t-\lim x_{k}=\xi$. Statistical convergence is a natural generalization of ordinary convergence. If $\lim x_{k}=$ $\xi$, then $s t-\lim x_{k}=\xi$. The converse does not hold in general.
Definition 1 (see [13]). A family of sets $I \subseteq 2^{\mathbb{N}}$ is called an ideal on $\mathbb{N}$ if and only if

(i) $\emptyset \in I$;

(ii) for each $A, B \in I$ one has $A \cup B \in I$;

(iii) for each $A \in I$ and each $B \subseteq A$ one has $B \in I$.

An ideal is called nontrivial if $\mathbb{N} \notin I$, and nontrivial ideal is called admissible if $\{n\} \in I$ for each $n \in \mathbb{N}$.

Definition 2 (see [14]). A family of sets $F \subseteq 2^{\mathbb{N}}$ is a filter in $\mathbb{N}$ if and only if

(i) $\emptyset \notin F$;

(ii) for each $A, B \in F$ one has $A \cap B \in F$;

(iii) for each $A \in F$ and each $B \supseteq A$ one has $B \in F$.

Proposition 3 (see [14]). I is a nontrivial ideal in $\mathbb{N}$ if and only if

$$
F=F(I)=\{M=\mathbb{N} \backslash A: A \in I\}
$$

is a filter in $\mathbb{N}$.

Definition 4 (see [14]). Let $I$ be a nontrivial ideal of subsets of $\mathbb{N}$. A number sequence $\left(x_{n}\right)_{n \in \mathbb{N}}$ is said to be $I$-convergent to $\xi$ $\left(\xi=I-\lim _{n \rightarrow \infty} x_{n}\right)$ if and only if for each $\varepsilon>0$ the set

$$
\left\{k \in \mathbb{N}:\left|x_{k}-\xi\right| \geq \varepsilon\right\}
$$

belongs to $I$. The element $\xi$ is called the $I$ limit of the number sequence $x=\left(x_{n}\right)_{n \in \mathbb{N}}$. 
The concept of $I$-convergence of real sequences is a generalization of statistical convergence which is based on the structure of the ideal $I$ of subsets of the set of natural numbers. Kostyrko et al. [14] introduced the concept of $I$ convergence of sequences in a metric space and studied some properties of this convergence. $I$-convergence of real sequences coincides with the ordinary convergence if $I$ is the ideal of all finite subsets of $\mathbb{N}$ and with the statistical convergence if $I$ is the ideal of subsets of $\mathbb{N}$ of natural density zero.

Definition 5 (see [14]). An admissible ideal $I \subseteq 2^{\mathbb{N}}$ is said to have the property (AP) if for any sequence $\left\{A_{1}, A_{2}, \ldots\right\}$ of mutually disjoint sets of $I$, there is sequence $\left\{B_{1}, B_{2}, \ldots\right\}$ of sets such that each symmetric difference $A_{i} \Delta B_{i}(i=1,2, \ldots)$ is finite and $\bigcup_{i=1}^{\infty} B_{i} \in I$.

Definition 5 is similar to the condition (APO) used in [15].

In [14], the concept of $I^{*}$-convergence which is closely related to $I$-convergence has been introduced.

Definition 6 (see [14]). A sequence $x=\left(x_{n}\right)$ of elements of $X$ is said to be $I^{*}$-convergence to $\xi$ if and only if there exists a set $M \in F(I)$,

$$
M=\left\{m=\left(m_{i}\right): m_{i}<m_{i+1}, i \in \mathbb{N}\right\} \subset \mathbb{N}
$$

such that $\lim _{k \rightarrow \infty} x_{m_{k}}=\xi$.

In [14], it is proved that $I$-convergence and $I^{*}$-convergence are equivalent for admissible ideals with property (AP).

Also, in order to prove that $I$-convergent sequence coincides with $I^{*}$-convergent sequence for admissible ideals with property (AP), we need the following lemma.

Lemma 7 (see [13]). Let $\left\{P_{i}\right\}_{i=1}^{\infty}$ be a countable collection of subsets of $\mathbb{N}$ such that $P_{i} \in F(I)$ is a filter which associates with an admissible ideal I with property (AP). Then there exists a set $P \subset \mathbb{N}$ such that $P \in F(I)$ and the set $P \backslash P_{i}$ is finite for all $i$.

Theorem 8 (see [13]). Let $I \subseteq 2^{\mathbb{N}}$ be an admissible ideals with property $(A P)$ and $x=\left(x_{n}\right)$ be a number sequence. Then I$\lim _{n \rightarrow \infty} x_{n}=\xi$ if and only if there exists a set $P \in F(I), P=$ $\left\{p=\left(p_{i}\right): p_{i}<p_{i+1}, \quad i \in \mathbb{N}\right\}$ such that $\lim _{k \rightarrow \infty} x_{p_{k}}=\xi$.

Definition 9 (see [9]). Let $(X, d)$ be a metric space. For any nonempty closed subsets $A, A_{k} \subseteq X$, one says that the sequence $\left\{A_{k}\right\}$ is Wijsman convergent to $A$ :

$$
\lim _{k \rightarrow \infty} d\left(x, A_{k}\right)=d(x, A)
$$

for each $x \in X$. In this case one writes $W-\lim _{k \rightarrow \infty} A_{k}=A$.

As an example, consider the following sequence of circles in the $(x, y)$-plane: $A_{k}=\left\{(x, y): x^{2}+y^{2}+2 k x=0\right\}$. As $k \rightarrow \infty$ the sequence is Wijsman convergent to the $y$-axis $A=\{(x, y): x=0\}$.

Definition 10 (see [16]). Let $(X, d)$ be a metric space. For any nonempty closed subsets $A, A_{k} \subseteq X$, one says that the sequence $\left\{A_{k}\right\}$ is Wijsman statistical convergent to $A$ if for $\varepsilon>0$ and for each $x \in X$,

$$
\lim _{n \rightarrow \infty} \frac{1}{n}\left|\left\{k \leq n:\left|d\left(x, A_{k}\right)-d(x, A)\right| \geq \varepsilon\right\}\right|=0 .
$$

In this case one writes $s t-\lim _{W} A_{k}=A$ or $A_{k} \rightarrow A(\mathrm{WS})$. Consider

$$
\text { WS }:=\left\{\left\{A_{k}\right\}: s t-\lim _{W} A_{k}=A\right\} \text {, }
$$

where WS denotes the set of Wijsman statistical convergence sequences.

Also the concept of bounded sequence for sequences of sets was given by Nuray and Rhoades [16] as follows.

Let $(X, \rho)$ be a metric space. For any nonempty closed subsets $A_{k}$ of $X$, one says that the sequence $\left\{A_{k}\right\}$ is bounded if $\sup _{k} d\left(x, A_{k}\right)<\infty$ for each $x \in X$.

\section{Wijsman $I$-Convergence}

In this section, we will define Wijsman $I$-convergence and Wijsman $I^{*}$-convergence of sequences of sets, give the relationship between them, and establish some basic theorems.

Definition 11. Let $(X, d)$ be a metric space and $I \subseteq 2^{\mathbb{N}}$ be a proper ideal in $\mathbb{N}$. For any nonempty closed subsets $A, A_{k} \subset$ $X$, one says that the sequence $\left\{A_{k}\right\}$ is Wijsman $I$-convergent to $A$, if, for each $\varepsilon>0$ and for each $x \in X$, the set

$$
A(x, \varepsilon)=\left\{k \in \mathbb{N}:\left|d\left(x, A_{k}\right)-d(x, A)\right| \geq \varepsilon\right\}
$$

belongs to $I$. In this case, one writes $I_{W}-\lim A_{k}=A$ or $A_{k} \rightarrow$ $A\left(I_{W}\right)$, and the set of Wijsman $I$-convergent sequences of sets will be denoted by

$$
I_{W}=\left\{\left\{A_{k}\right\}:\left\{k \in \mathbb{N}:\left|d\left(x, A_{k}\right)-d(x, A)\right| \geq \varepsilon\right\} \in I\right\} .
$$

Example 12. $I \subseteq 2^{\mathbb{N}}$ be a proper ideal in $\mathbb{N},(X, d)$ a metric space, and $A, A_{k} \subset X$ nonempty closed subsets. Let $X=\mathbb{R}^{2}$, $\left\{A_{k}\right\}$ be following sequence:

$$
\begin{gathered}
A_{k}=\left\{\begin{array}{cc}
\left\{(x, y) \in \mathbb{R}^{2}: x^{2}+y^{2}-2 k y=0\right\} & \text { if, } k \neq n^{2} \\
\left\{(x, y) \in \mathbb{R}^{2}: y=-1\right\} & \text { if, } k=n^{2},
\end{array}\right. \\
A=\left\{(x, y) \in \mathbb{R}^{2}: y=0\right\} .
\end{gathered}
$$

For $k=n^{2}, d\left((x, y), A_{n^{2}}\right)=|y+1| \neq d((x, y) ; A)=|y|$. Let us take a point $\left(x^{*}, y^{*}\right)$ outside $x^{2}+y^{2}-2 k y=0$. For $k \neq n^{2}$, we write $d\left(\left(x^{*}, y^{*}\right), A_{k}\right) \rightarrow d\left(\left(x^{*}, y^{*}\right), A\right)=\left|y^{*}\right|$. Since the line equation is

$$
\frac{x-0}{x^{*}}=\frac{y-k}{y^{*}-k},
$$

where the line is passing from $(0, k)$ the center point of the circle and $\left(x^{*}, y^{*}\right)$ the outside of the circle, we write $y=k+$ 
$\left(\left(y^{*}-k\right) / x^{*}\right) \cdot x$. If we write this $y=k+\left(\left(y^{*}-k\right) / x^{*}\right) \cdot x$ value on the circle equation $x^{2}+y^{2}-2 k y=0$, we can get

$$
x=\frac{|k| \cdot x^{*}}{\sqrt{\left(x^{*}\right)^{2}+\left(y^{*}-k\right)^{2}}} .
$$

For $k \rightarrow \infty$, if we take limit, it will be $x \rightarrow x^{*}$. If we write $x=\left(|k| \cdot x^{*}\right) / \sqrt{\left(x^{*}\right)^{2}+\left(y^{*}-k\right)^{2}}$ on the $y=k+\left(\left(y^{*}-k\right) / x^{*}\right)$. $x$, we get $y \rightarrow 0(k \rightarrow \infty)$. Thus, for $k \neq n^{2}$

$$
d\left(\left(x^{*}, y^{*}\right), A_{k}\right)=\sqrt{\left(x-x^{*}\right)^{2}+\left(y-y^{*}\right)^{2}} \longrightarrow\left|y^{*}\right|
$$

So we get $d\left(\left(x^{*}, y^{*}\right), A_{k}\right) \rightarrow d\left(\left(x^{*}, y^{*}\right), A\right)=\left|y^{*}\right|$, for $k \neq n^{2}$.

For $k=n^{2}$ and $k \neq n^{2}$, the set sequence $\left\{A_{k}\right\}$ has two different limits. Thus $\left\{A_{k}\right\}$ is not Wijsman convergent to set $A$, but

$$
\begin{gathered}
\left\{k \in \mathbb{N}:\left|d\left((x, y), A_{k}\right)-d((x, y), A)\right| \geq \varepsilon\right\} \\
=\left\{k \in \mathbb{N}: k=n^{2}\right\} \subset I_{d} .
\end{gathered}
$$

Thus, suppose that

$$
A(x, y, \varepsilon)=\left\{k \in \mathbb{N}:\left|d\left((x, y), A_{k}\right)-d((x, y), A)\right| \geq \varepsilon\right\}
$$

for $\varepsilon>0$ and for each $(x, y) \in \mathbb{R}^{2}$.

Since $\lim _{k \rightarrow \infty}\left[\left|d\left((x, y), A_{k}\right)-d((x, y), A)\right|\right]=0$, for $k \neq n^{2}$, for each $\varepsilon>0$,

$$
\exists k_{\varepsilon} \in \mathbb{N}: \forall k>k_{\varepsilon}:\left|d\left((x, y), A_{k}\right)-d((x, y), A)\right|<\varepsilon .
$$

Define the set $A_{k_{\varepsilon}}(x, y)$ as

$$
A_{k_{\varepsilon}}(x, y):=\left\{k \in \mathbb{N}:\left|d\left((x, y), A_{k}\right)-d((x, y), A)\right|>\varepsilon\right\} .
$$

Thus, since $A(x, y, \varepsilon)=A_{k_{\varepsilon}}(x, y) \cup\left\{k \in \mathbb{N}: k=n^{2}\right\}$ and $A_{k_{\varepsilon}}(x, y) \in I_{d}$ and $\left\{k \in \mathbb{N}: k=n^{2}\right\} \in I_{d}$, we can write

$$
\begin{aligned}
& A(x, y, \varepsilon) \\
& \quad:=\left\{k \in \mathbb{N}:\left|d\left((x, y), A_{k}\right)-d((x, y), A)\right|>\varepsilon\right\} \in I_{d},
\end{aligned}
$$

where $I_{d}=\{A: \delta(A)=0\}$. So the set sequence $\left\{A_{n}\right\}$ is Wijsman $I$-convergent to set $A$.

Example 13. Let $I \subseteq 2^{\mathbb{N}}$ be a proper ideal in $\mathbb{N},(X, d)$ a metric space, and $A, A_{n} \subset X$ nonempty closed subsets. Let $X=\mathbb{R}^{2}$, $\left\{A_{n}\right\}$ be following sequence:

$$
\begin{gathered}
A_{n} \\
= \begin{cases}\left\{(x, y) \in \mathbb{R}^{2}: 0 \leq x \leq n, 0 \leq y \leq \frac{1}{n} \cdot x\right\}, & \text { if, } n \neq k^{2} \\
\left\{(x, y) \in \mathbb{R}^{2}: x \geq 0, y=1\right\}, & \text { if, } n=k^{2},\end{cases} \\
A=\left\{(x, y) \in \mathbb{R}^{2}: x \geq 0, y=0\right\} .
\end{gathered}
$$

Since

$$
\lim _{n \rightarrow \infty} \frac{1}{n}\left|\left\{k \leq n:\left|d\left((x, y), A_{n}\right)-d((x, y), A)\right| \geq \varepsilon\right\}\right|=0,
$$

the set sequence $\left\{A_{n}\right\}$ is Wijsman statistical convergent to set $A$. Thus we can write st $-\lim _{W} A_{n}=A$, but this sequence is not Wijsman convergent to set $A$. Because for $n \neq k^{2}, \lim _{n \rightarrow \infty} d\left((x, y), A_{n}\right)=d((x, y), A)$, but for $n=k^{2}$, $\lim _{n \rightarrow \infty} d\left((x, y), A_{n}\right) \neq d((x, y), A)$. Let $I_{d} \subset 2^{\mathbb{N}}$ be proper ideal. Define set $K$ as

$$
K=K(\varepsilon)=\left\{n \in \mathbb{N}:\left|d\left((x, y), A_{n}\right)-d((x, y), A)\right| \geq \varepsilon\right\} .
$$

If we take $I_{d}$ for $I$, Wijsman ideal convergent coincides with Wijsman statistical convergent. Really, one has

$$
\begin{gathered}
\left\{n \in \mathbb{N}:\left|d\left((x, y), A_{n}\right)-d((x, y), A)\right| \geq \varepsilon\right\} \\
=\left\{n \in \mathbb{N}: n=k^{2}\right\} \subset I_{d} .
\end{gathered}
$$

Since the Wijsman topology is not first countable in general, if $\left\{A_{k}\right\}$ is convergent to the set $A$ Wijsman sense, every subsequence of $\left\{A_{k}\right\}$ may not be convergent to $A$. But if $X$ is separable, then every subsequence of a convergent set sequence is convergent to the same limit.

Definition 14. Let $I \subseteq 2^{\mathbb{N}}$ be a proper ideal in $\mathbb{N}$ and $(X, d)$ be a separable metric space. For any nonempty closed subsets $A, A_{k} \subset X$, one says that the sequence $\left\{A_{k}\right\}$ is Wijsman $I^{*}$-convergent to $A$, if and only if there exists a set $M \in$ $F(I), M=\left\{m=\left(m_{i}\right): m_{i}<m_{i+1}, i \in \mathbb{N}\right\} \subset \mathbb{N}$ such that for each $x \in X$

$$
\lim _{k \rightarrow \infty} d\left(x, A_{m_{k}}\right)=d(x, A) .
$$

In this case, one writes $I_{W}^{*}-\lim A_{k}=A$.

Definition 15. Let $I \subseteq 2^{\mathbb{N}}$ be an admissible ideal in $\mathbb{N}$ and $(X, d)$ be a separable metric space. For any nonempty closed subset $A_{n}$ in $X$, one says that the sequence $\left\{A_{n}\right\}$ is Wijsman $I$-Cauchy sequence if for each $\varepsilon>0$ and for each $x \in X$, there exists a number $N=N(\varepsilon)$ such that

$$
\left\{n \in \mathbb{N}:\left|d\left(x, A_{n}\right)-d\left(x, A_{N}\right)\right| \geq \varepsilon\right\}
$$

belongs to $I$.

Definition 16. Let $I \subseteq 2^{\mathbb{N}}$ be an admissible ideal in $\mathbb{N}$ and $(X, d)$ be a separable metric space. For any nonempty closed subsets $A_{k} \subset X$, one says that the sequence $\left\{A_{k}\right\}$ is Wijsman $I^{*}$-Cauchy sequences if there exists a set $M=\left\{m=\left(m_{i}\right)\right.$ : $\left.m_{i}<m_{i+1}, \quad i \in \mathbb{N}\right\} \subset \mathbb{N}, M \in F(I)$ such that the subsequence $A_{M}=\left\{A_{m_{k}}\right\}$ is Wijsman Cauchy in $X$; that is,

$$
\lim _{k, p \rightarrow \infty}\left|d\left(x, A_{m_{k}}\right)-d\left(x, A_{m_{p}}\right)\right|=0
$$

Now we will prove that Wijsman $I$-convergence implies the Wijsman I-Cauchy condition. 
Theorem 17. Let I be an arbitrary admissible ideal and let $X$ be a separable metric space. Then $I_{W}-\lim A_{n}=A$ implies that $\left\{A_{n}\right\}$ is Wijsman I-Cauchy sequence.

Proof. Let $I$ be an arbitrary admissible ideal and $I_{W}-\lim A_{n}=$ $A$. Then for each $\varepsilon>0$ and for each $x \in X$, we have

$$
A(x, \varepsilon)=\left\{n \in \mathbb{N}:\left|d\left(x, A_{n}\right)-d(x, A)\right| \geq \varepsilon\right\}
$$

that belongs to $I$. Since $I$ is an admissible ideal, there exists an $n_{0} \in \mathbb{N}$ such that $n_{0} \notin A(x, \varepsilon)$.

Let $B(x, \varepsilon)=\left\{n \in \mathbb{N}:\left|d\left(x, A_{n}\right)-d\left(x, A_{n_{0}}\right)\right| \geq 2 \varepsilon\right\}$. Taking into account the inequality

$$
\begin{aligned}
& \left|d\left(x, A_{n}\right)-d\left(x, A_{n_{0}}\right)\right| \\
& \quad \leq\left|d\left(x, A_{n}\right)-d(x, A)\right|+\left|d\left(x, A_{n_{0}}\right)-d(x, A)\right|,
\end{aligned}
$$

we observe that if $n \in B(x, \varepsilon)$, then

$$
\left|d\left(x, A_{n}\right)-d(x, A)\right|+\left|d\left(x, A_{n_{0}}\right)-d(x, A)\right| \geq 2 \varepsilon .
$$

On the other hand, since $n_{0} \notin A(x, \varepsilon)$, we have $\mid d\left(x, A_{n_{0}}\right)-$ $d(x, A) \mid<\varepsilon$. Here we conclude that $\left|d\left(x, A_{n}\right)-d(x, A)\right| \geq \varepsilon$; hence $n \in A(x, \varepsilon)$. Observe that $B(x, \varepsilon) \subset A(x, \varepsilon) \in I$ for each $\varepsilon>0$ and for each $x \in X$. This gives that $B(x, \varepsilon) \in I$; that is $\left\{A_{n}\right\}$ is Wijsman $I$-Cauchy sequence.

Theorem 18. Let $I$ be an admissible ideal and let $X$ be a separable metric space. If $\left\{A_{n}\right\}$ is Wijsman $I^{*}$-Cauchy sequence, then it is Wijsman I-Cauchy sequence.

Proof. Let $\left\{A_{n}\right\}$ be Wijsman $I^{*}$-Cauchy sequence; then by the definition, there exists a set $M=\left\{m=\left(m_{i}\right): m_{i}<m_{i+1}, i \in\right.$ $\mathbb{N}\} \subset \mathbb{N}, M \in F(I)$ such that

$$
\left|d\left(x, A_{m_{k}}\right)-d\left(x, A_{m_{p}}\right)\right|<\varepsilon
$$

for each $\varepsilon>0$, for each $x \in X$, and for all $k, p>k_{0}=k_{0}(\varepsilon)$.

Let $N=N(\varepsilon)=m_{k_{0}+1}$. Then for every $\varepsilon>0$, we have

$$
\left|d\left(x, A_{m_{k}}\right)-d\left(x, A_{N}\right)\right|<\varepsilon, \quad k>k_{0} .
$$

Now let $H=\mathbb{N} \backslash M$. It is clear that $H \in I$ and that

$$
\begin{aligned}
A(x, \varepsilon)= & \left\{n \in \mathbb{N}:\left|d\left(x, A_{n}\right)-d\left(x, A_{N}\right)\right| \geq \varepsilon\right\} \\
& \subset H \cup\left\{m_{1}, m_{2}, \ldots, m_{k_{0}}\right\}
\end{aligned}
$$

belongs to $I$. Therefore, for every $\varepsilon>0$, we can find a $N=$ $N(\varepsilon)$ such that $A(x, \varepsilon) \in I$; that is, $\left\{A_{n}\right\}$ is Wijsman I-Cauchy sequence. Hence the proof is complete.

In order to prove that Wijsman $I$-convergent sequence coincides with Wijsman $I^{*}$-convergent sequence for admissible ideals with property (AP), we need the following lemma.

Lemma 19. Let $I \subseteq 2^{\mathbb{N}}$ be an admissible ideal with property $(A P)$ and $(X, d)$ a separable metric space. If $I_{W}-$ $\lim _{n \rightarrow \infty} d\left(x, A_{n}\right)=d(x, A)$, then there exists a set $P \in$ $F(I) P=\left\{p=\left(p_{i}\right): p_{i}<p_{i+1}, i \in \mathbb{N}\right\}$ such that $I_{W^{-}}$ $\lim _{k \rightarrow \infty} d\left(x, A_{p_{k}}\right)=d(x, A)$.
Theorem 20. Let $I \subseteq 2^{\mathbb{N}}$ be an admissible ideal with property $(A P)$, let $(X, d)$ be an arbitrary separable metric space and $x=$ $\left(x_{n}\right) \in X$. Then, $I_{W}-\lim _{n \rightarrow \infty} d\left(x, A_{n}\right)=d(x, A)$, if and only if there exists a set $P \in F(I), P=\left\{p=\left(p_{i}\right): p_{i}<p_{i+1}, i \in \mathbb{N}\right\}$ such that $I_{W}-\lim _{k \rightarrow \infty} d\left(x, A_{p_{k}}\right)=d(x, A)$.

Now we prove that, a Wijsman I-Cauchy sequence coincides with a Wijsman $I^{*}$-Cauchy sequence for admissible ideals with property (AP).

Theorem 21. If $I \subseteq 2^{\mathbb{N}}$ is an admissible ideal with property $(A P)$ and if $(X, d)$ is a separable metric space, then the concepts Wijsman I-Cauchy sequence and Wijsman I* -Cauchy sequence coincide.

Proof. If a sequence is Wijsman $I^{*}$-Cauchy, then it is Wijsman $I$-Cauchy by Theorem 18 where $I$ does not need to have the (AP) property. Now it is sufficient to prove that $\left\{A_{n}\right\}$ is Wijsman $I^{*}$-Cauchy sequence in $X$ under assumption that $\left\{A_{n}\right\}$ is a Wijsman $I$-Cauchy sequence. Let $\left\{A_{n}\right\}$ be a Wijsman $I$-Cauchy sequence. Then by definition, there exists a $N=$ $N(\varepsilon)$ such that

$$
A(x, \varepsilon)=\left\{n \in \mathbb{N}:\left|d\left(x, A_{n}\right)-d\left(x, A_{N}\right)\right| \geq \varepsilon\right\} \in I
$$

for each $\varepsilon>0$ and for each $x \in X$.

Let $P_{i}=\left\{n \in \mathbb{N}:\left|d\left(x, A_{n}\right)-d\left(x, A_{m_{i}}\right)\right|<1 / i\right\}, i=1,2, \ldots$ where $m_{i}=N(1 / i)$. It is clear that $P_{i} \in F(I)$ for $i=1,2, \ldots$. Since $I$ has (AP) property, then by Lemma 7 there exists a set $P \subset \mathbb{N}$ such that $P \in F(I)$ and $P \backslash P_{i}$ is finite for all $i$. Now we show that

$$
\lim _{n, m \rightarrow \infty}\left|d\left(x, A_{n}\right)-d\left(x, A_{m}\right)\right|=0 .
$$

To prove this, let $\varepsilon>0, x \in X$, and $j \in \mathbb{N}$ such that $j>2 / \varepsilon$. If $m, n \in P$ then $P \backslash P_{i}$ is finite set, therefore there exists $k=k(j)$ such that

$$
\begin{aligned}
& \left|d\left(x, A_{n}\right)-d\left(x, A_{m_{j}}\right)\right|<\frac{1}{j}, \\
& \left|d\left(x, A_{m}\right)-d\left(x, A_{m_{j}}\right)\right|<\frac{1}{j}
\end{aligned}
$$

for all $m, n>k(j)$. Hence it follows that

$$
\begin{aligned}
\left|d\left(x, A_{n}\right)-d\left(x, A_{m}\right)\right|< & \left|d\left(x, A_{n}\right)-d\left(x, A_{m_{j}}\right)\right| \\
& +\left|d\left(x, A_{m}\right)-d\left(x, A_{m_{j}}\right)\right|<\varepsilon
\end{aligned}
$$

for $m, n>k(j)$. $F(I)$ :

Thus, for any $\varepsilon>0$, there exists $k=k(\varepsilon)$ and $n, m \in P \in$

$$
\left|d\left(x, A_{n}\right)-d\left(x, A_{m}\right)\right|<\varepsilon .
$$

This shows that the sequences $\left\{A_{n}\right\}$ is a Wijsman $I^{*}$-Cauchy sequence. 
Theorem 22. Let $I$ be an admissible ideal and $(X, d) a$ separable metric space. Then $I_{W}^{*}-\lim A_{k}=A$ implies that $\left\{A_{n}\right\}$ is a Wijsman I-Cauchy sequence.

Proof. Let $I_{W}^{*}-\lim A_{k}=A$. Then by definition there exists a set $M \in F(I), M=\left\{m=\left(m_{i}\right): m_{i}<m_{i+1}, i \in \mathbb{N}\right\} \subset \mathbb{N}$ such that

$$
\lim _{k \rightarrow \infty} d\left(x, A_{m_{k}}\right)=d(x, A)
$$

for each $\varepsilon>0$ and for each $x \in X$, and $k, p>k_{0}$,

$$
\begin{aligned}
& \left|d\left(x, A_{m_{k}}\right)-d\left(x, A_{m_{p}}\right)\right| \\
& \quad<\left|d\left(x, A_{m_{k}}\right)-d(x, A)\right|+\left|d\left(x, A_{m_{p}}\right)-d(x, A)\right| \\
& \quad<\frac{\varepsilon}{2}+\frac{\varepsilon}{2}=\varepsilon .
\end{aligned}
$$

Therefore,

$$
\lim _{k, p \rightarrow \infty}\left|d\left(x, A_{m_{k}}\right)-d\left(x, A_{m_{p}}\right)\right|=0 .
$$

Hence, $\left\{A_{n}\right\}$ is a Wijsman I-Cauchy sequence.

Theorem 23. Let $I$ be an admissible ideal and $(X, d) a$ separable metric space. If the ideal I has property $(A P)$ and if $(X, d)$ is an arbitrary metric space, then for arbitrary sequence $\left\{A_{n}\right\}_{n \in \mathbb{N}}$ of elements of $X I_{W}-\lim A_{n}=A$ implies $I_{W}^{*}-$ $\lim A_{n}=A$.

Proof. Suppose that $I$ satisfies condition (AP). Let $I_{W}-$ $\lim A_{n}=A$. Then

$$
T(\varepsilon, x)=\left\{n \in \mathbb{N}:\left|d\left(x, A_{n}\right)-d(x, A)\right| \geq \varepsilon\right\} \in I
$$

for each $\varepsilon>0$ and for each $x \in X$. Put

$$
\begin{gathered}
T_{1}=\left\{n \in \mathbb{N}:\left|d\left(x, A_{n}\right)-d(x, A)\right| \geq 1\right\}, \\
T_{n}=\left\{n \in \mathbb{N}: \frac{1}{n} \leq\left|d\left(x, A_{n}\right)-d(x, A)\right|<\frac{1}{n-1}\right\}
\end{gathered}
$$

for $n \geq 2$, and $n \in \mathbb{N}$. Obviously $T_{i} \cap T_{j}=\emptyset$ for $i \neq j$. By condition (AP) there exists a sequence of sets $\left\{V_{n}\right\}_{n \in \mathbb{N}}$ such that $T_{j} \Delta V_{j}$ are finite sets for $j \in \mathbb{N}$ and $V=\bigcup_{j=1}^{\infty} V_{j} \in I$. It is sufficient to prove that for $M=\mathbb{N} \backslash V, M=\left\{m=\left(m_{i}\right): m_{i}<\right.$ $\left.m_{i+1}, \quad i \in \mathbb{N}\right\} \in F(I)$, we have $\lim _{k \rightarrow \infty} d\left(x, A_{m_{k}}\right)=d(x, A)$.

Let $\gamma>0$. Choose $k \in \mathbb{N}$ such that $1 /(k+1)<\gamma$. Then

$$
\left\{n \in \mathbb{N}:\left|d\left(x, A_{n}\right)-d(x, A)\right| \geq \gamma\right\} \subset \bigcup_{j=1}^{k+1} T_{j} .
$$

Since $T_{j} \Delta V_{j}, j=1,2, \ldots$ are finite sets, there exists $n_{0} \in \mathbb{N}$ such that

$$
\begin{aligned}
& \left(\bigcup_{j=1}^{k+1} V_{j}\right) \cap\left\{n \in \mathbb{N}: n>n_{0}\right\} \\
& \quad=\left(\bigcup_{j=1}^{k+1} T_{j}\right) \cap\left\{n \in \mathbb{N}: n>n_{0}\right\} .
\end{aligned}
$$

If $n>n_{0}$ and $n \notin V$, so $n \notin \bigcup_{j=1}^{k+1} V_{j}$ and by (44) $n \notin \bigcup_{j=1}^{k+1} T_{j}$. But then $\left|d\left(x, A_{n}\right)-d(x, A)\right|<1 /(n+1)<\gamma$ for each $x \in X$, so we have $\lim _{k \rightarrow \infty} d\left(x, A_{m_{k}}\right)=d(x, A)$.

\section{Wijsman $I$-Limit Points and Wijsman $I$-Cluster Points Sequences of Sets}

In this section, we introduce Wijsman I-limit points of sequences of sets and Wijsman $I$-cluster points of sequences of sets, prove some basic properties of these concepts, and establish some basic theorems.

Definition 24. Let $I \subseteq 2^{\mathbb{N}}$ a proper ideal in $\mathbb{N}$ and $(X, d)$ a separable metric space. For any nonempty closed subsets $A_{n}$, $B_{n} \subset X$, one says that the sequences $\left\{A_{n}\right\}$ and $\left\{B_{n}\right\}$ are almost equal with respect to $I$ if

$$
\left\{n \in \mathbb{N}: A_{n} \neq B_{n}\right\} \in I
$$

and we write $I$-a.a.n $A_{n}=B_{n}$.

Definition 25. Let $I \subseteq 2^{\mathbb{N}}$ be a proper ideal in $\mathbb{N}$ and let $(X, d)$ be a separable metric space; $A_{n}$ is nonempty closed subset of $X$. If $\left\{A_{n}\right\}_{K}$ is subsequence of $\left\{A_{n}\right\}$ and $K:=\{n(j): j \in$ $\mathbb{N}\}$, then we abbreviate $\left\{A_{n_{j}}\right\}$ by $\left\{A_{n}\right\}_{K}$. If $K \in I$, then $\left\{A_{n}\right\}_{K}$ subsequence is called thin subsequence of $\left\{A_{n}\right\}$. If $K \notin I$, then $\left\{A_{n}\right\}_{K}$ subsequence is called nonthin subsequence of $\left\{A_{n}\right\}$.

Definition 26. Let $I \subseteq 2^{\mathbb{N}}$ be a proper ideal in $\mathbb{N}$ and let $(X, d)$ be a separable metric space, for any nonempty closed subsets $A_{k} \subset X$. One has the following.

(i) $A \in X$ is said to be a Wijsman $I$-limit point of $\left\{A_{n}\right\}$ provided that there is a set $M=\left\{m=\left(m_{i}\right): m_{i}<\right.$ $\left.m_{i+1}, i \in \mathbb{N}\right\} \subset \mathbb{N}$ such that $M \notin I$ and for each $x \in$ $X \lim _{k \rightarrow \infty} d\left(x, A_{m_{k}}\right)=d(x, A)$.

(ii) $A \in X$ is said to be a Wijsman $I$-cluster point of $\left\{A_{n}\right\}$ if and only if for each $\varepsilon>0$, for each $x \in X$, we have

$$
\left\{n \in \mathbb{N}:\left|d\left(x, A_{n}\right)-d(x, A)\right|<\varepsilon\right\} \notin I .
$$

Denote by $I_{W}\left(\Lambda_{\left\{A_{n}\right\}}\right), I_{W}\left(\Gamma_{\left\{A_{n}\right\}}\right)$, and $L_{\left\{A_{n}\right\}}$ the set of all Wijsman $I$-limit, Wijsman $I$-cluster, and Wijsman limit points of $\left\{A_{n}\right\}$, respectively.

For the sequences $\left\{A_{n}\right\}, I_{W}\left(\Gamma_{\left\{A_{n}\right\}}\right) \subseteq I_{W}\left(L_{\left\{A_{n}\right\}}\right)$. Let $A \in$ $I_{W}\left(\Gamma_{\left\{A_{n}\right\}}\right)$. Then for each sequence $\left\{A_{n}\right\} \subset X$, we have $\lim _{k \rightarrow \infty} d\left(x, A_{m_{k}}\right)=d(x, A)$ which means that $A \in L_{\left\{A_{n}\right\}}$.

Theorem 27. Let $I \subseteq 2^{\mathbb{N}}$ be a proper ideal in $\mathbb{N}$ and let $(X, d)$ be a separable metric space. Then for each sequence $\left\{A_{n}\right\} \subset X$ one has $I_{W}\left(\Lambda_{\left\{A_{n}\right\}}\right) \subset I_{W}\left(\Gamma_{\left\{A_{n}\right\}}\right)$.

Proof. Let $A \in I_{W}\left(\Lambda_{\left\{A_{n}\right\}}\right)$. Then, there exists $M=\left\{m_{1}<\right.$ $\left.m_{2}<\cdots\right\} \subset \mathbb{N}$ such that $M=\left\{m=\left(m_{i}\right): m_{i}<m_{i+1}, i \in\right.$ $\mathbb{N}\} \notin I$ and

$$
\lim _{k \rightarrow \infty} d\left(x, A_{m_{k}}\right)=d(x, A)
$$


According to (47), there exists $k_{0} \in \mathbb{N}$ such that for each $\varepsilon>0$, for each $x \in X$ and $k>k_{0},\left|d\left(x, A_{m_{k}}\right)-d(x, A)\right|<\varepsilon$. Hence,

$$
\begin{gathered}
\left\{k \in \mathbb{N}:\left|d\left(x, A_{m_{k}}\right)-d(x, A)\right|<\varepsilon\right\} \\
\supseteq M \backslash\left\{m_{1}, m_{2}, \ldots, m_{k_{o}}\right\} .
\end{gathered}
$$

Then, the set on the right hand side of (48) does not belong to $I$; therefore

$$
\left\{k \in \mathbb{N}:\left|d\left(x, A_{m_{k}}\right)-d(x, A)\right|<\varepsilon\right\} \notin I
$$

which means that $A \in I_{W}\left(\Gamma_{\left\{A_{n}\right\}}\right)$.

Theorem 28. Let $I \subseteq 2^{\mathbb{N}}$ be a proper ideal in $\mathbb{N}$ and let $(X, d)$ be a separable metric space. Then for each sequence $\left\{A_{n}\right\} \subset X$ one has $I_{W}\left(\Gamma_{\left\{A_{n}\right\}}\right) \subseteq L_{\left\{A_{n}\right\}}$.

Proof. Let $A \in I_{W}\left(\Gamma_{\left\{A_{n}\right\}}\right)$. Then for each $\varepsilon>0$ and for each $x \in X$, we have

$$
\left\{n \in \mathbb{N}:\left|d\left(x, A_{n}\right)-d(x, A)\right|<\varepsilon\right\} \notin I .
$$

Let

$$
K_{n}:=\left\{n \in \mathbb{N}:\left|d\left(x, A_{n}\right)-d(x, A)\right|<\frac{1}{n}\right\}
$$

for $n \in \mathbb{N}$. $\left\{K_{n}\right\}_{n=1}^{\infty}$ is decreasing sequence of infinite subsets of $\mathbb{N}$. Hence $K=\left\{n=\left(n_{i}\right): n_{i}<n_{i+1}, i \in \mathbb{N}\right\} \notin I$ such that $\lim _{n \rightarrow \infty} d\left(x, A_{n_{i}}\right)=d(x, A)$ which means that $A \in L_{\left\{A_{n}\right\}}$.

Theorem 29. Let $I \subseteq 2^{\mathbb{N}}$ a proper ideal in $\mathbb{N},(X, d)$ a separable metric space, and $A_{k}, B_{k}$ nonempty subsets of $X$. If $\left\{A_{k}\right\}=$ $\left\{B_{k}\right\}$ I-a.a.k for $k \in \mathbb{N}$, then $I_{W}\left(\Gamma_{\left\{A_{k}\right\}}\right)=I_{W}\left(\Gamma_{\left\{B_{k}\right\}}\right)$ and $I_{W}\left(\Lambda_{\left\{A_{k}\right\}}\right)=I_{W}\left(\Lambda_{\left\{B_{k}\right\}}\right)$.

Proof. If $\left\{A_{k}\right\}=\left\{B_{k}\right\}$ a.a.k for $k \in \mathbb{N}$, then

$$
K:=\left\{k \in \mathbb{N}: A_{k} \neq B_{k}\right\} \in I
$$

Let $A \in I_{W}\left(\Gamma_{\left\{A_{k}\right\}}\right)$. For each $\varepsilon>0$ and for each $x \in X$ we have

$$
\left\{k \in \mathbb{N}:\left|d\left(x, A_{k}\right)-d(x, A)\right|<\varepsilon\right\} \notin I,
$$

$\forall \varepsilon>0$. If $\left\{A_{k}\right\}=\left\{B_{k}\right\} I$-a.a.k, then $\left\{k \in \mathbb{N}: \mid d\left(x, B_{k}\right)-\right.$ $d(x, A) \mid<\varepsilon\} \notin I$ which means that $A \in I_{W}\left(\Gamma_{\left\{B_{k}\right\}}\right)$; hence $I_{W}\left(\Gamma_{\left\{A_{k}\right\}} \subset I_{W}\left(\Gamma_{\left\{B_{k}\right\}}\right)\right.$. Similarly we can also prove that $I_{W}\left(\Gamma_{\left\{B_{k}\right\}}\right) \subset I_{W}\left(\Gamma_{\left\{A_{k}\right\}}\right.$. So we have $I_{W}\left(\Gamma_{\left\{A_{k}\right\}}=I_{W}\left(\Gamma_{\left\{B_{k}\right\}}\right)\right.$.

Now, we show that $I_{W}\left(\Lambda_{\left\{A_{k}\right\}}\right)=I_{W}\left(\Lambda_{\left\{B_{k}\right\}}\right)$. Let $A \in$ $I_{W}\left(\Lambda_{\left\{A_{k}\right\}}\right)$. Then there exists a set $M=\left\{m=\left(m_{i}\right): m_{i}<\right.$ $\left.m_{i+1}, i \in \mathbb{N}\right\} \subset \mathbb{N}$ such that $M \notin I$ and

$$
\begin{gathered}
\lim _{k \rightarrow \infty} d\left(x, A_{m_{k}}\right)=d(x, A), \\
M=\left\{k: k \in M \text { and } A_{k} \neq B_{k}\right\} \\
\cup\left\{k: k \in M \text { and } A_{k}=B_{k}\right\},
\end{gathered}
$$

$M \notin I$, and hence $\left\{k: k \in M\right.$ and $\left.A_{k}=B_{k}\right\} \notin I$. Then there exists

$$
P=\left\{p=\left(p_{i}\right): p_{i}<p_{i+1}, i \in \mathbb{N}\right\} \notin I
$$

such that

$$
\lim _{k \rightarrow \infty} d\left(x, B_{p_{k}}\right)=d(x, A)
$$

which means that $A \in I_{W}\left(\Lambda_{\left\{B_{k}\right\}}\right)$. Similarly we can also prove that $I_{W}\left(\Lambda_{\left\{B_{k}\right\}}\right) \subset I_{W}\left(\Lambda_{\left\{A_{k}\right\}}\right)$. Therefore we have $I_{W}\left(\Lambda_{\left\{A_{k}\right\}}\right)=$ $I_{W}\left(\Lambda_{\left\{B_{k}\right\}}\right)$.

\section{References}

[1] J.-P. Aubin and H. Frankowska, Set-Valued Analysis, vol. 2, Birkhäuser, Boston, Mass, USA, 1990.

[2] M. Baronti and P. L. Papini, "Convergence of sequences of sets," in Methods of Functional Analysis in Approximation Theory, vol. 76, pp. 135-155, Birkhäuser, Basel, Switzerland, 1986.

[3] G. Beer, "Convergence of continuous linear functionals and their level sets," Archiv der Mathematik, vol. 52, no. 5, pp. 482491, 1989.

[4] G. Beer, "On convergence of closed sets in a metric space and distance functions," Bulletin of the Australian Mathematical Society, vol. 31, no. 3, pp. 421-432, 1985.

[5] J. Borwein and J. Vanderwerff, "Dual Kadec-Klee norms and the relationships between Wijsman, slice, and Mosco convergence," The Michigan Mathematical Journal, vol. 41, no. 2, pp. 371-387, 1994.

[6] O. Kisi and F. Nuray, "On $S_{\lambda}^{L}$ (I)-asymptotically statistical equivalence of sequences of sets," Mathematical Analysis, vol. 2013, Article ID 602963, 6 pages, 2013.

[7] Y. Sonntag and C. Zălinescu, "Set convergences: an attempt of classification," Transactions of the American Mathematical Society, vol. 340, no. 1, pp. 199-226, 1993.

[8] R. A. Wijsman, "Convergence of sequences of convex sets, cones and functions," Bulletin of the American Mathematical Society, vol. 70, pp. 186-188, 1964.

[9] R. A. Wijsman, "Convergence of sequences of convex sets, cones and functions. II," Transactions of the American Mathematical Society, vol. 123, pp. 32-45, 1966.

[10] R. C. Buck, "Generalized asymptotic density," The American Journal of Mathematics, vol. 75, pp. 335-346, 1953.

[11] H. Fast, "Sur la convergence statistique," Colloquium Mathematicae, vol. 2, pp. 241-244, 1951.

[12] I. J. Schoenberg, "The integrability of certain functions and related summability methods," The American Mathematical Monthly, vol. 66, pp. 361-375, 1959.

[13] P. Kostyrko, M. Mačaj, T. Šalát, and M. Sleziak, "I-convergence and extremal I-limit points," Mathematica Slovaca, vol. 55, no. 4, pp. 443-464, 2005.

[14] P. Kostyrko, T. Šalát, and W. Wilczyński, “I-convergence,” Real Analysis Exchange, vol. 26, no. 2, pp. 669-685, 2000.

[15] A. R. Freedman and J. J. Sember, "Densities and summability," Pacific Journal of Mathematics, vol. 95, no. 2, pp. 293-305, 1981.

[16] F. Nuray and B. E. Rhoades, "Statistical convergence of sequences of sets," Fasciculi Mathematici, no. 49, pp. 87-99, 2012. 


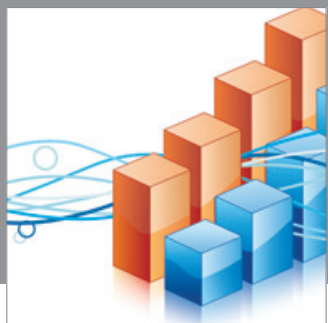

Advances in

Operations Research

mansans

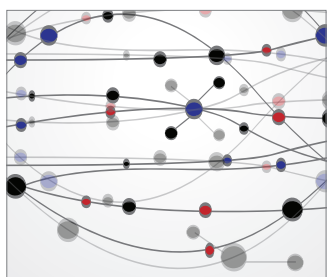

The Scientific World Journal
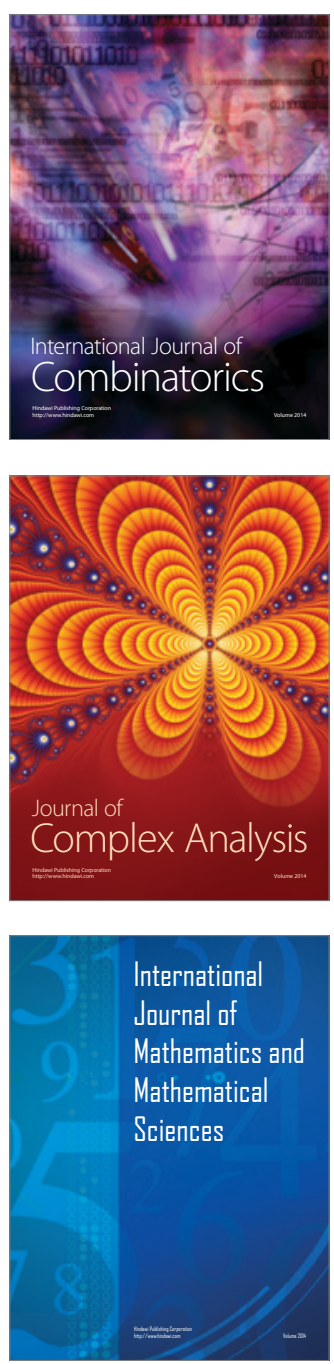
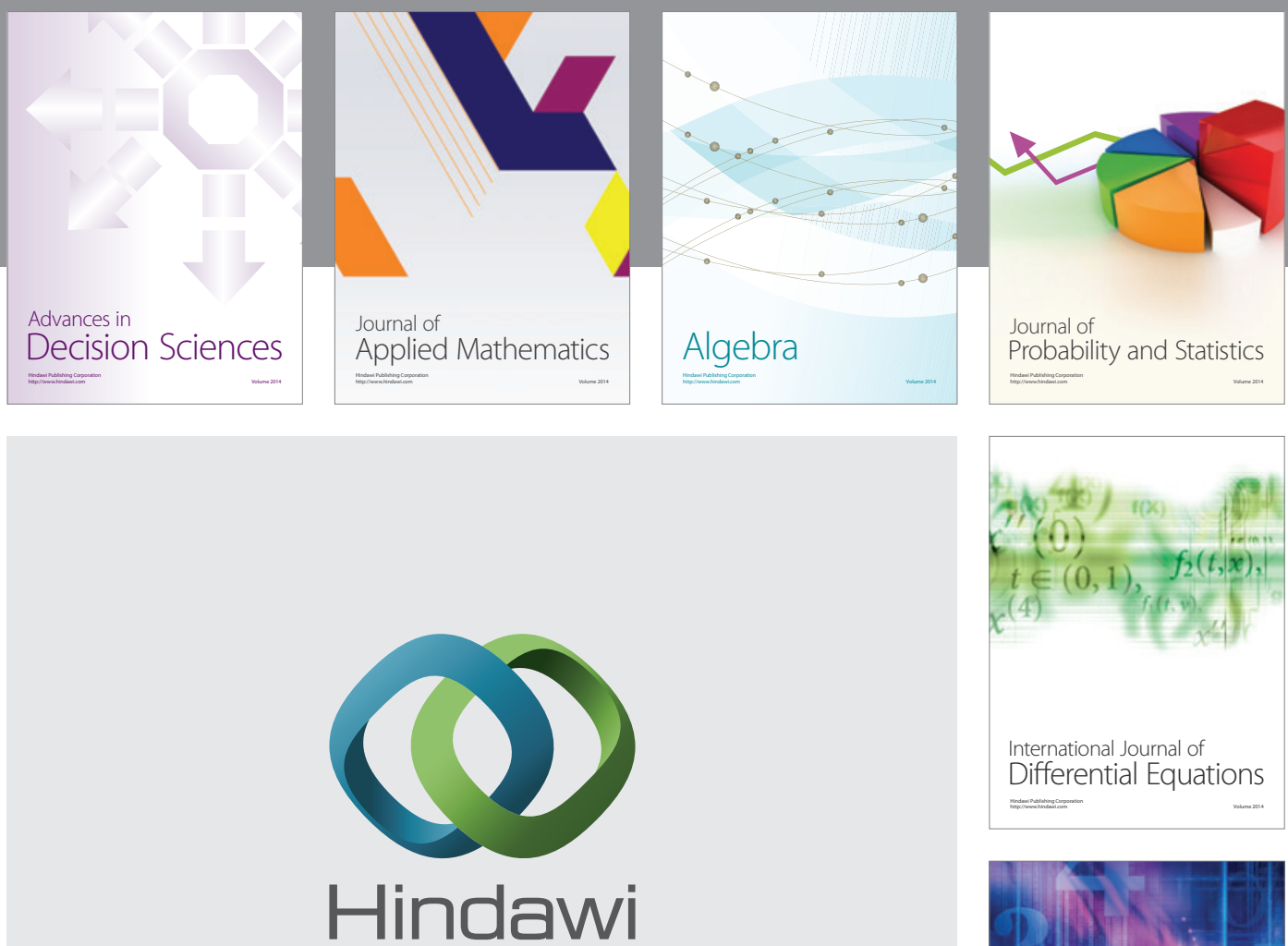

Submit your manuscripts at http://www.hindawi.com
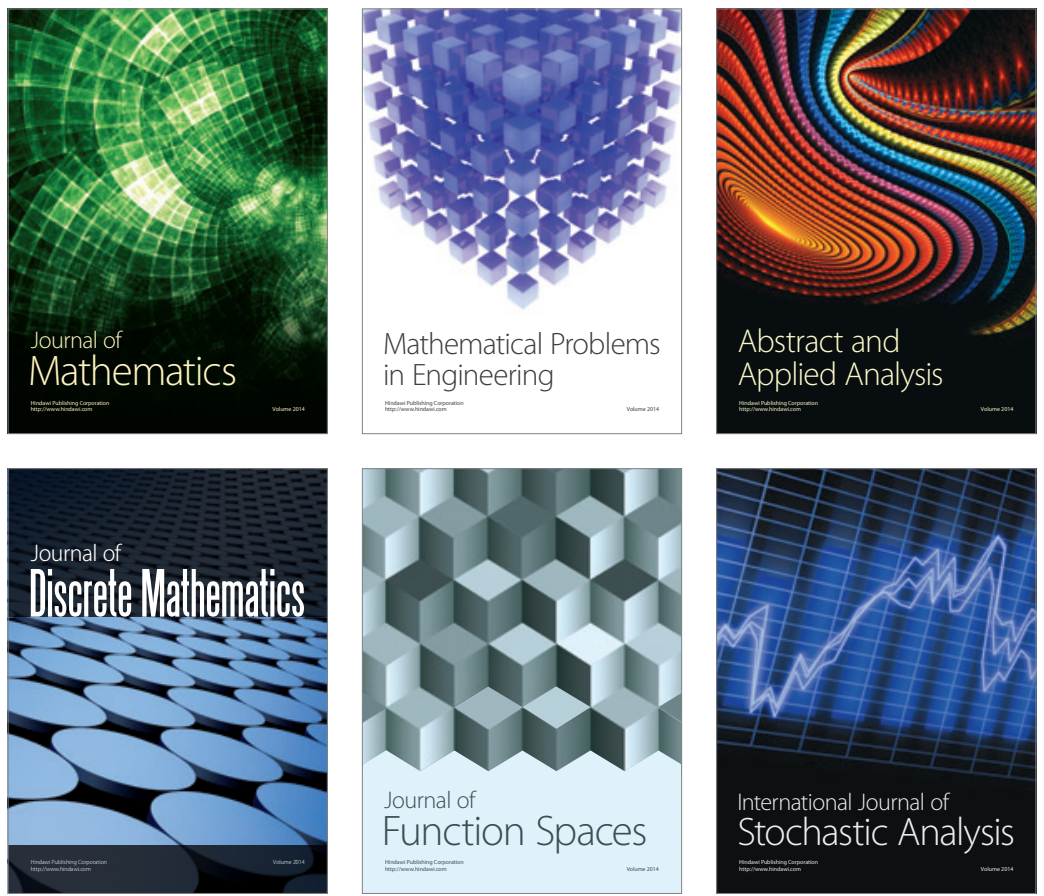

Journal of

Function Spaces

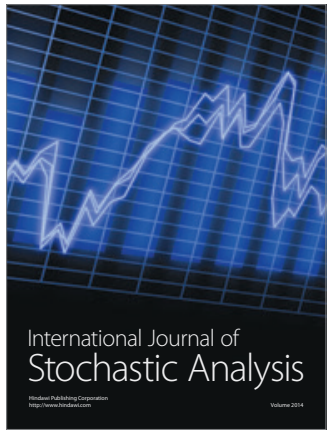

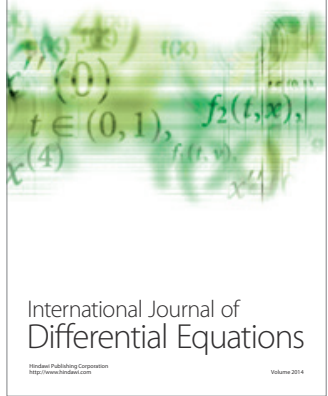
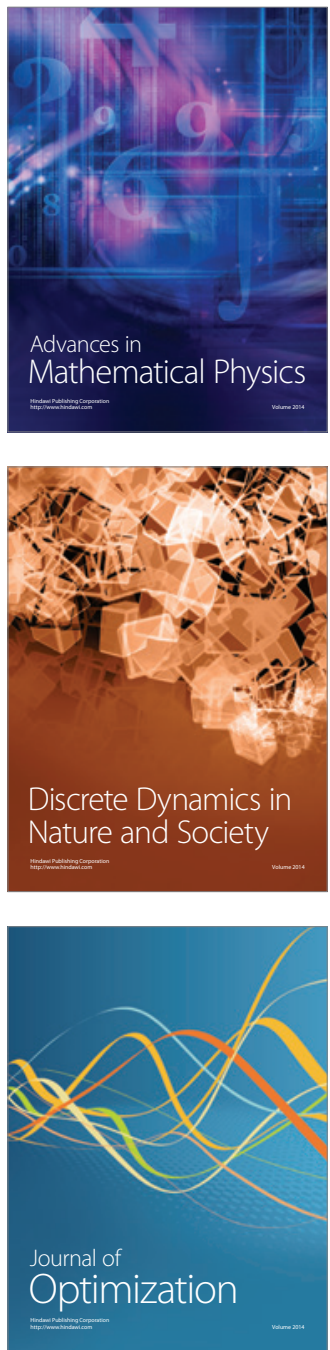